Rev. salud pública. 10 (1):94-104, 2008

\title{
Demora en el Diagnóstico de Tuberculosis Pulmonar en una región de Colombia
}

\author{
Delayed diagnosis of pulmonary tuberculosis in a particular \\ part of Colombia
}

\author{
Flor de María Cáceres-Manrique y Luís C. Orozco-Vargas
}

Departamento de Salud Pública, Escuela de Medicina y Escuela de Enfermería, Facultad de Salud, Universidad Industrial de Santander, Bucaramanga, Colombia. fmcacer@uis.edu.co,

flordemar200@yahoo.com,lcorovar@uis.edu.co

Recibido 5 Marzo 2007/Enviado para Modificación 13 Noviembre 2007/Aceptado 15 Diciembre 2007

\section{RESUMEN}

Objetivo Determinar la demora en el diagnóstico de tuberculosis y los factores asociados a la misma.

Métodos. Estudio de Corte transversal, mediante entrevista a tuberculosos adultos, se obtuvieron variables sociodemográficas y tiempo desde el inicio de síntomas al diagnóstico. Se definió como demora el tiempo mayor a la mediana del intervalo, así: demora del paciente, entre inicio de síntomas y primera consulta; demora de servicios de salud, desde la primera consulta hasta el diagnóstico; $y$, demora total del inicio de síntomas al diagnóstico. Se calcularon OR para establecer asociación entre demora y varios factores mediante modelos de regresión logística.

Resultados Se estudiaron 216 participantes, $61 \%$ hombres y con edad promedio de 43 años. La demora del paciente fue mayor a 30 días y estuvo asociada a desempleo (OR 2.56, IC95 \% 1,28-4,76) y no tener seguridad social (OR 2,32, IC95\% 1,20-4,50). La demora de servicios de salud fue mayor a 60 días y estuvo asociada al régimen contributivo de seguridad social (OR 1,91, IC95 \% 1,07-3,44) y al desplazamiento (OR 0,20 , IC95 \% 0,06-0,67). La demora total fue de 120 días asociada a no tener seguridad social (OR 3,54, IC95 \% 2,25-10,8).

Conclusión La mediana del tiempo total entre el inicio de síntomas y el diagnóstico de tuberculosis fue de 120 días. Otros estudios reportan entre 42 y 119 días. Se recomienda agilizar el diagnóstico de tuberculosis pulmonar para evitar el contagio a otras personas. Se requieren reforzar el compromiso de los servicios de salud.

Palabras Clave: Tuberculosis, diagnóstico, remisión y consulta, servicios de salud. (source: DeCS, BIREME).

\section{ABSTRACT}

Objective The present study was aimed at establishing delay in diagnosing tuberculosis and associated factors.

Methods This was a cross-sectional study. Adults from the city of Bucaramanga proving smear-positive for tuberculosis were interviewed for establishing demographic and 
social variables; the onset of symptoms, the date of their first visit to a doctor and time of diagnosis were established. Delay was defined as being any time longer than the mathematical average interval for such attention. Patient delay was taken as being from the onset of symptoms to the first visit to a doctor. Health service delay consisted of the time taken from first visit to diagnosis. Total delay was regarded as being from the onset of symptoms until diagnosis. Odds ratios (OR) were calculated and association between delay and risk factors was evaluated by using logistical regression.

Results Two hundred and sixteen patients were interviewed; 131 (61\%) were males and mean age was 43. Patient delay was longer than 30 days; it was associated with unemployment (2.56 OR; 1.28-4.76 $95 \% \mathrm{Cl})$ and the absence of social security $(2.32$ OR; 1.20-4.50 $95 \% \mathrm{Cl}$ ). Health service delay was greater than 60 days and was associated with the contributive social security regime (1.91 OR; 1.07-3.44 $95 \% \mathrm{Cl}$ ) and displacement $(0.20 \mathrm{OR}$; 0.06-0.67 $95 \% \mathrm{Cl})$. Total delay was greater than 120 days and was associated with patients lacking social security (3.54 OR; 2.25-10.8 $95 \% \mathrm{Cl}$ ). Conclusion Average delay time for diagnosing pulmonary tuberculosis was 120 days. This was higher than in other studies which have reported delay as being 42 to 119 days. We recommend expediting the diagnosis of pulmonary tuberculosis so that other people do not become infected and improving health service commitment.

Key Words: tuberculosis, diagnosis, delay, health service (source: $M e S H, N L M$ ).

$\mathrm{E}$ l diagnóstico temprano de la tuberculosis (TBC) y el inicio oportuno del tratamiento son esenciales para lograr un control efectivo de la enfermedad (1). La demora en el diagnóstico e inicio del tratamiento es uno de losprimerosobstáculos para el programa y un problema muy importante de salud pública. Se estima que por cada año que pasa un paciente con TBC pulmonar, sin recibir tratamiento, puede llegar a infectar entre $10 \mathrm{y}$ 15 personas más (2). La proporción de contactos que se infectan a partir de un caso positivo pueden llegar a ser entre un 30 a 40 \% (1). La demora conlleva mayor deterioro de la salud del paciente e incrementa la infectividad en la comunidad (3), con lo cual se aumenta la morbimortalidad y la transmisión de la infección (4).

Llegar al diagnóstico de TBC es una responsabilidad compartida. En primer lugar, es del paciente, quien debe acudir oportunamente a consulta médica. En segundo lugar, del médico, quien debe sospechar la enfermedad, ordenar las pruebas correspondientes, informar al paciente sobre los pasos a seguir para confirmar el diagnóstico y educarlo sobre la importancia de seguir las recomendaciones. Por último, de los servicios de salud, que deben realizar las pruebas y orientar al paciente de acuerdo al resultado (5). El diagnóstico involucra un compromiso para comenzar y terminar el tratamiento (6). 
Se han estudiado varios factores asociados a la demora en el diagnóstico $(7,8)$, pero en nuestro medio no se conoce el comportamiento del fenómeno ni los factores asociados para dirigir las intervenciones. El presente estudio tiene como objetivo determinar el tiempo de demora en el diagnóstico de TBC pulmonar y la asociación del mismo con edad, género, tipo de seguridad social, conocimientos sobre la TBC, distancia de su domicilio a la institución de salud, estrato socioeconómico y nivel educativo de los pacientes procedentes de un área metropolitana de Colombia, que incluye Bucaramanga, la ciudad capital, y los municipios aledaños: Girón, Floridablanca y Piedecuesta.

\section{MÉTODOS}

Este es un estudio de corte transversal, aprobado por el Comité de Ética de la Universidad Industrial de Santander (UIS). El estudio se llevó a cabo en la ciudad de Bucaramanga y los municipios de Girón, Floridablanca y Piedecuesta, que concentran más del 80 \% de la población del Departamento de Santander, Colombia. A todos los pacientes mayores de 15 años que ingresaron a los programas de TBC, con diagnóstico de TBC pulmonar, entre el $1^{\circ}$ de junio del 2002 y el 31 de mayo del 2003, previo consentimiento informado, se les realizó una entrevista con una encuesta estructurada. La entrevista se llevó a cabo durante los primeros 15 días del ingreso del paciente al programa de TBC, en la Institución Prestadora de Servicios de Salud (IPS) donde se encontraba recibiendo el tratamiento. Se interrogó por el momento de inicio de los síntomas (tos con expectoración) y el tiempo transcurrido entre el inicio de los síntomas, la primera consulta médica y la fecha del diagnóstico. Se averiguó además por otras variables como edad, raza, sexo, escolaridad, estado civil, convivencia, empleo, tenencia de vivienda, estrato socioeconómico de ubicación de la vivienda, condición de desplazamiento, conocimientos sobre TBC, distancia a los centros de salud y tipo de seguridad social. De la tarjeta de medicamentos existente en el programa se tomó el dato de fecha de diagnóstico de TBC pulmonar. Se excluyeron aquellos pacientes quienes no fue posible contactarlos para la entrevista en los primeros 15 días de inicio del tratamiento.

Se consideró que presentaba demora todo paciente cuyos tiempos fueran superiores a la mediana de las distribución de los tiempos en cada uno de los intervalos de la siguiente manera:: demora del paciente(DP) tiempo transcurrido entre el inicio de los síntomas y la primera consulta a un médico; demora de los servicios de salud (DSS), tiempo entre la primera consulta y el diagnóstico de TBC; y, demora total (DT) tiempo entre el inicio de los síntomas y el diagnóstico. 
Se elaboró una base de datos en Epi-info 6.03b (9), que se importo a Stata 8.0 (10), programa en el cual se realizó el análisis de los datos. Se calcularon las medidas de tendencia central de cada uno de los tiempos: media (prom), mediana (me), percentil 25 (p25) y percentil 75 (p75), así como las medianas de los tiempos para establecer la demora. Una vez determinadas las medianas se generaron las variables dicotómicas de cada uno de los tipos de demora (DP, DSS y DT) y se distribuyeron los participantes según si presentaban o no demora.

Se evaluó la asociación entre la demora y cada una de las variables del paciente, el tratamiento y los servicios de salud. Aquellas asociaciones cuyas probabilidades de error tipo I (p) fueron menores de 0,2 se introdujeron en un modelo de regresión logística, paso a paso, siguiendo las recomendaciones de Greenland (11) para evaluar su asociación con los diferentes tipos de demora, controlando por las demás variables, usando regresión logística. Se probó la bondad de ajuste de los modelos siguiendo la técnica de Hosmer y Lemeshow (12).

\section{RESULTADOS}

Ingresaron 221 pacientes con TBC pulmonar al programa de TBC durante el periodo de estudio, de los cuales se encuestaron 216. Los otros cinco (2,3\%) no fue posible entrevistarlos en los primeros 15 días de inicio del tratamiento, quedando 216 para el análisis.

De los 216 participantes, 131 (61 \%) eran hombres. La edad estaba entre 15 y 96 años con promedio de 43 y mediana de 40 años. La escolaridad estuvo entre 0 y 17 años aprobados, con promedio de 6 y mediana de 5 años. Un total de 87 (40 \%) estaban solteros, 95 (44 \%) eran casados o vivían en unión libre y 34 (16\%) eran viudos o estaban separados. 22 pacientes (10 \%) vivían solos y 34 (16 \%) manifestaron no contar con apoyo familiar para llevar a cabo su tratamiento. En cuanto a la vivienda, 98 (45 \%) habitaban en vivienda propia, 81 (38 \%) en viviendas arrendadas y 37 (17\%) pacientes era otro tipo de tenencia de la vivienda donde residían. Un total de 71 pacientes (33\%) tenían empleo y 19 (8,8 \%) eran desplazados. Un total de 77 participantes (36 \%) pertenecían al régimen contributivo del Sistema de Seguridad Social en Salud, 41 (19 \%) al subsidiado, 73 (34 \%) estaban vinculados y 24 (11 \%) no tenían ningún tipo de seguridad social. 
La DT fue de 45,1 días y está compuesta por 15,8 (35 \%) días que transcurrieron entre el inicio de síntomas y la primera consulta al médico, demora del paciente (DP) y 29.370 (65 \%) días desde la primera consulta al diagnóstico (DSS). La distribución por cuarteles de los tiempos entre inicio de síntomas, primera consulta y diagnóstico, se presentan en la Tabla 1.

Tabla 1. Distribución de los tiempos en días desde el inicio de los síntomas hasta el diagnóstico de TBC pulmonar

\begin{tabular}{lccc}
\hline \multicolumn{1}{c}{ Tiempo } & P25 & Med & P75 \\
\hline Del inicio de sintomas a la primera consulta & 15 & 30 & 90 \\
De la primera consulta al diagnóstico & 15 & 60 & 150 \\
Desde el inicio de sintomas al diagnóstico & 60 & 120 & 240 \\
\hline P25: percentil 25: Med: mediana; P75: percentil 75 & & &
\end{tabular}

La mediana del tiempo entre el inicio de los síntomas y la primera consulta fue de 30 días; por esta razón, 140 pacientes (64,8 \%) que habían demorado más de un mes en consultar se les consideró que tenían DP.

La mediana entre la primera consulta y el diagnóstico fue de 60 días, por lo tanto los 114 pacientes (52,8 \%) que demoraron mayor tiempo se consideró que tenían DSS. La mediana del tiempo entre el inicio de los síntomas y el diagnóstico fue de 120 días (4 meses): 96 (44,4\%) personas con tiempo mayor a éste se consideró que tenían DT.

La DP estuvo independientemente asociada al desempleo (OR 2,54 IC95 \% 1,36-4,74), ser mayor de 20 años (5,30, IC95\% 1,51-18,67), no tener seguridad social (OR 2,11, IC95 \% 1,14-3,89) y en el límite de la significancia con ser hombre (OR 1,81, IC95 \% 0,98-3,32). En la Tabla 2 se presenta el modelo de regresión logística que muestra estas asociaciones.

Tabla 2. Factores asociados a la demora del paciente, por empleo, edad, sexo y seguridad social.

\begin{tabular}{lccc}
\hline \multicolumn{1}{c}{ Variable } & OR & IC 95 \% & P \\
\hline Desempleo & 2,54 & $1,36-4,74$ & 0,003 \\
Edad > de 20años & 5,30 & $1,51-18,67$ & 0,009 \\
No tener Seguridad Social & 2,11 & $1,14-3,89$ & 0,017 \\
Sexo masculino & 1,81 & $0,98-3,32$ & 0,057 \\
\hline Bondad de ajuste de Hosmer y Lemeshow: $\mathrm{X}^{2}=7,45 ; \mathrm{p}=0,591$ &
\end{tabular}

La DSS estuvo asociada a ser desplazado como factor protector (OR 0,21, IC95 \% 0,06-0,68), y pertenecer al régimen contributivo de seguridad social (OR 2,00, IC95 \% 1,06-3,70), haber presentado DP (OR 2,06, IC95 \% 1,103,86 ) y tener entre 20 y 29 años o entre 60 y 69 años de edad, como factores 
de riesgo. En la Tabla 3 se presenta el modelo de regresión logística que evalúan la asociación entre la DSS por cada una de las variables.

La DT estuvo asociada con la falta de seguridad social (OR 3,54, IC95 \% 2,25-10,8).

Tabla 3. Factores asociados a la demora de los servicios de salud,

\begin{tabular}{lccc}
\multicolumn{4}{c}{ controlando por edad y sexo } \\
\hline \multicolumn{1}{c}{ Variable } & OR & IC 95 \% & P \\
\hline Ser desplazado & 0,21 & $0,06-0,68$ & 0,010 \\
Régimen contributivo & 2,00 & $1,06-3,70$ & 0,033 \\
Haber presentado DP & 2,06 & $1,10-3,86$ & 0,024 \\
Edad 20-29 ó 60-69 años & 2,17 & $1,18-4,01$ & 0,013 \\
Sexo masculino & 1,07 & $0,59-1,95$ & 0,809 \\
Bondad de ajuste de Hosmer y Lemeshow: $\mathrm{X}^{2}=31,0 ; \mathrm{p}=0,745$
\end{tabular}

\section{DISCUSIÓN}

Auque se espera que en ninguna parte del mundo y bajo ninguna circunstancia haya demora en el diagnóstico de la TBC, dado que los programas tienen normas claras sobre búsqueda, diagnóstico, tratamiento y seguimiento, que han probado ser eficaces, las diferencias entre los países es difícil de valorar ya que numerosos factores personales y culturales intervenienen, así como la diversidad de sistemas de salud y procesos administrativos.

La demora en el diagnóstico de la TBC es frecuente y prolongada en nuestro medio. La mediana de la DT fue de 120 días (4 meses), tiempo en el cual el paciente está sufriendo un deterioro en su salud y está infectando a más personas. Llama la atención que los tiempos de demora encontrados en el presente estudio están por encima de lo informado en la literatura. Por ejemplo, en estudios realizados en Canadá, Malasia y Ghana, la DT presentó una mediana de 42, 88 y 119 días, respectivamente (3). En Malawi la mediana de la DT fue de 56 días (13), mientras que en New York fue de 57 días (14); en Londres de 49 días (4) y en la India de 60 días (15). Mientras que un estudio informó una mediana de DT igual al presente informe (4 meses) (16) y otro presenta medianas de DT mayores; es el caso de un estudio en Tanzania (17), en el cual se reportaron medianas de DT de 136 días. Es posible que estas diferencias se deban a la manera como están organizado el sistema de salud, a la descentralización del programa de TBC y a la disminución en la búsqueda de sintomáticos respiratorios $(18,19)$. 
Un 35 \% del tiempo de DT corresponde a DP y el 65 \% a DSS. En la mayoría de los estudios informan este mismo comportamiento (15,20-25); sin embargo, hay un estudio que encontró que el 80 \% de la DT era del paciente (26), mientras que en otro se encontró que la mediana de DP era de 25 días y la DSS de 15 días (14). Esto habla de la accesibilidad que las personas tienen a los servicios de salud y su adecuada respuesta a las necesidades de las personas.

La DSS se podría explicar por los trámites que exige el sistema para prestar la atención (27) y por la distancia entre los sitios de consulta, los laboratorios clínicos y de rayos $\mathrm{X}$. Es posible que ante esto el paciente desista y tome la decisión de automedicarse para aliviar los síntomas. Un estudio encontró que los costos, la baja calidad de los servicios de salud y las actitudes del personal de salud están asociados a esta demora (28).

En los países donde el sistema de salud es responsabilidad directa del Estado definen como demora del médico lo que en este estudio se ha definido como DSS; es más, atribuyen la DSS al mal entrenamiento de los médicos y proponen la educación continuada y supervisión de los mismos como medida para disminuir la demora (29). En este estudio no es posible diferenciar la demora debida al quehacer del médico de la demora ocasionada por otros miembros del equipo de salud y por los procesos administrativos.

No hubo diferencias por sexo en la demora en el diagnóstico de la TBC pulmonar. Algunos estudios han encontrado que la DT en mujeres es mayor que en hombres, diferencias explicadas porque hay inequidad en la atención de las mujeres; allí, a pesar que las mujeres consultaban más temprano, la DP en mujeres fue el doble que en hombres (de 41 vs 19 días) (30). Esos hallazgos fueron similares a dos estudios, uno llevado a cabo en Nepal (31) y otro en Londres (4).

No hubo diferencias en la demora entre los pacientes por nivel de conocimiento, como si los muestra el estudio de Nigeria (32); puede ser porque en el presente estudio el nivel de conocimiento se midió cuando el paciente ya había iniciado del tratamiento y en este momento pudo haber mejorado su nivel de conocimiento con respecto a la TBC. La escolaridad de las personas, el estado civil, la percepción sobre apoyo familiar, la percepción de la distancia al Centro de Salud y tener otras patologías tampoco mostró asociación con la demora en el diagnostico de TBC. 
Una de las fortalezas del presente estudio consiste en que es el primero que trata de caracterizar la demora en el diagnóstico de TBC en nuestro medio, después de implementada la Ley 100 de Seguridad Social y la descentralización del programa de TBC. Lo anterior hizo que todas las responsabilidades fueran delegadas del Ministerio de Salud (2), hoy Ministerio de la Protección Social hacia las Empresas Promotoras de Salud (EPS) y las Administradoras del Régimen Subsidiado (ARS). Esto conlleva que el proceso de diagnóstico sea contratado con laboratorios ubicados en sitios distantes al lugar de la consulta, lo cual constituye una barrera de acceso adicional (19).

Este estudio puede tener varias limitaciones; entre ellas, el tratarse de un estudio de corte transversal limita la posibilidad de establecer la relación causa efecto. La medida de la variable de salida, tiempo de demora fue por auto reporte y estaba sujeta al recuerdo del paciente por lo cual pudo llevar a sesgo de clasificación, además del evidente sesgo de dígito (15 días, un mes, seis meses, un año). Por otro lado, se hizo énfasis en los aspectos del paciente y no en lo concerniente a los Servicios de Salud.

El hecho que las encuestas fuesen realizadas en el centro de salud o la IPS a donde el paciente acude puede haber interferido con la veracidad de las respuestas del paciente, pues puede haber presentido que al decir la verdad sobre la demora en la prestación de los servicios deteriorara la calidad del servicio que se le estaba prestando. Se requiere hacer nuevas investigación con énfasis en los aspectos relacionados con la DSS.

La entrevista no se pudo realizar a 5 (2,3 \%) ya que no se logró entrevista oportuna en los primeros 15 días de tratamiento. Sin embargo estos pacientes eran muy similares en edad, género y nivel educativo con los participantes en el estudio, por lo que es muy poco probable que haya sesgo de selección.

En conclusión, la demora en el diagnóstico de la TBC es común y prolongada en nuestro medio. Es superior a los hallazgos en otros países y va en contra de lo que se considera la primera medida de prevención de la enfermedad, que es detectar tempranamente los casos e iniciarles tratamiento (2). Se recomienda afiliar a todos las personas al Sistema de Seguridad Social, involucrar a los servicios de salud y motivarlos para enfatizar en la búsqueda de TBC entre sintomáticos respiratorios, acelerar los procesos para realizar el diagnóstico temprano e iniciar el tratamiento cuando antes * 
Agradecimientos. Trabajo financiado por la Universidad Industrial de Santander y el Instituto Colombiano para el Desarrollo de la Ciencia y la Tecnología "Francisco José de Caldas" (Colciencias), (Contrato No. 101 - 2003; Código 1102-04-12907).

Conflictos de interés: ninguno

\section{REFERENCIAS}

1. Demisse M, Lindtjorn B, Berhane Y. Patient and health service delay in the diagnosis of pulmonary tuberculosis in Ethiopia. BMC Public Health 2002; 2:23.

2. República de Colombia, Ministerio de Salud. Guía de atención de la tuberculosis pulmonar y extrapulmonar. Bogotá, Ministerio de Salud, 2000.

3. Needham DM, Foster SD, Tomlinson G, Godfrey-Faussett. Socio-economic, gender and health services factors affecting diagnostic delay for tuberculosis patients in urban Zambia. Trop Med Intern Health 2001; 6:256-9.

4.Rodger A, Jaffar S, Paynter S, Hayward A, Carless J, Maguire H. Delay in the diagnosis of pulmonary tuberculosis, London, 1998-2000: analysis of surveillance data. $\mathrm{Br}$ Med J2003; 326:909-10.

5. Altet ML, Alcaide J, Canela J, Milá C, Jiménez ML, Solsona J. Estudio del retraso diagnóstico de la tuberculosis pulmonar sintomática. Arch Bronconeumol 2003; 39:146-52

6. Packham S. Tuberculosis in the elderly. Gerontology 2001; 47:175-9.

7. Bialik R. La adherencia terapéutica desde una perspectiva antropológica. Arch Neurocien 2003; 46-51.

8. Morales-Aguirre JJ, Coria-Lorenzo JJ, Ornelas-Carsolio ME, Gómez-Barreto D. Recomendaciones sobre el tratamiento de infecciones por Mycobacterium tuberculosis. Bol Med Hosp Infant Mex 2003; 60:111-24.

9. Dean J, Dean DA, Coloumbier D, Brebdel KA, Smith DC, Burton AH, et al. Epi Info 6.04c. A word processing, database, and statistic program for public health. Center for Disease Control and Prevention (Atlanta, USA), and World Health Organization. Geneva, Switzerland; 1997.

10. STATA 8.0. STATA Corporation, Inc., College Station, Texas, USA; 2003.

11. Greenland S. Modeling and variable selection in epidemiologic analysis. Am J Public Health 1989; 79:340-9.

12. Hosmer DW, Lemeshow S. Applied logistic regression. 2nd edition. New York: John Wiley \& Sons Inc; 2000.

13. Salaniponi FM, Harries AD, Banda HT, Kanglombe C, Mphasa N, Mwale A, et al. Care seeking behaviour and diagnostic processes in patients with smear-positive pulmonary tuberculosis in Malawi.It J Tuberc Lung Dis 2000; 4:327-32. 
14. Sherman LF, Fujiwara PI, Cook SV, Bazerman LB, Frieden TR. Patient and health care system delays in the diagnosis and treatment of tuberculosis. Int J Tuberc Lung Dis 1999; 3:1088-95.

15. Rajeswari R, Chandrasekaran V, Suhadev M, Sivasubramanian S, Sudha G, Renu G. Factors associated with patient and health system delays in the diagnosis of tuberculosis in South India. Int J Tuberc Lung Dis 2002; 6:789-95.

16. Lawn SD, Afful B, Acheampong JW Pulmonary tuberculosis: diagnostic delay in Ghanaian adults. Int J Tuberc Lung Dis 1998; 2:635-40.

17. Wandwalo ER, Morkve O. Delay in tuberculosis case-finding and treatment in Mwanza, Tanzania. Int J Tuberc Lung Dis 2000; 4:133-8.

18. Victoria JE. Epidemiología de la tuberculosis en Colombia. Inf Quinc Epidemiol Nac 1999; 4:82-5.

19. Arbelaez MP, Gaviria MB, Franco A, Restrepo R, Hincapie D, Blas E. Tuberculosis control and managed competition in Colombia. Int J HeaLth Plann Mgmt 2004; 19: s25-S43.

20. Sadiq H, MuynckAD Health care seeking behavior of pulmonary tuberculosis patients visiting TB Center Rawalpindi. Int J Tuberc Lung Dis 2002; 6:780-8

21. Calder L, Gao W, Simmons G.Tuberculosis: reasons for diagnostic delay in Auckland. NZ Med J 2000; 113:483-5.

22. Franco J, Blanquer R, Flores J, Fernández E, Plaza P, Nogueira JM. Análisis del retraso diagnóstico en tuberculosis. Med Clin (Barc) 1996;12:453-7.

23. Liam CK, Tang BG. Delay in the diagnosis and treatment of pulmonary tuberculosis in patients attending a university teaching hospital. Int J Tuberc Lung Dis 1997; 1:326-32.

24. Beyers N, Gie RP, Schaaf HS, Van Zyl S, Nel ED, Talent JM, et al. Delay in the diagnosis, notification and initiation of treatment and compliance in children with tuberculosis. Tuber Lung Dis 1994; 75:260-5.

25. Sasaki Y, Yamagishi F, Yagi T, Yamatani H, Kuroda F, Shoda H [Internet]. A study of patient's and doctor's delay in patients with pulmonary tuberculosis discovered by visiting doctors with symptoms in particular on doctor's delay. Kekkaku (Tuberculosis) 2000; 75:527-32. (Resumen en inglés de artículo en japonés. Disponible en: http://www.ncbi.nlm.nih.gov/sites/entrez?cmd=Retrieve \&db=PubMed\&list_uids=11068369\&dopt=Citation Consultado en febrero de 2007).

26. Mori T, Shimao T,Jin BW, Kim SJ. Analysis of case-finding process of tuberculosis in Korea. Tuber Lung Dis 1992; 73:225-31.

27. Asch S, Leake B, Anderson R, Gelberg L. Why do symptomatic patients delay obtaining care for tuberculosis? Am J Respir Care Med 1998; 157:1244-8. 
104 REVISTADE SALUD PÚBLICA · Volumen 10 (1), Febrero 2008

28. Johansson E, Long NH, Diwan VK, Winkvist A. Gender and tuberculosis control: perspectives on health seeking behavior among men and women in Vietnam. Health Policy 2000; 52:33-51.

29. Moro ML, Resi D, Mezzetti F, Borrini BM. Diagnostic delay in patients with pulmonary tuberculosis. Recenti Prog Med 2003; 94:157-62.

30. Thorson A, Hoa NP, Long NH. Health-seeking behavior of individuals with a cough of more than 3 weeks. Lancet 2000; 356:1823-4.

31. Yamasaki-Nakagawa M, Ozasa K, Yamada N, Osuga K, Shimouchi A, Ishikawa N, et al. Gender difference in delays to diagnosis and health care seeking behavior in a rural area of Nepal. Int J Tuberc Lung Dis 2001; 5:24-31.

32. Enwuru CA, Idigbe EO, Ezeobi NV, Otegbeye AF. Care-seeking behavioural patterns, awareness and diagnostic processes in patients with smear- and culture-positive pulmonary tuberculosis in Lagos, Nigeria. Trans R Soc Trop Med Hyg 2002; 96:614-6. 